\title{
CURRENT FUNDAMENTAL SYSTEM ERRORS AND POST-HIPPARCOS UTILIZATION OF GROUND-BASED OPTICAL INSTRUMENTS
}

\author{
G.A. GONCHAROV \\ Pulkovo Observatory \\ 196140, St.Petersburg, Russia
}

Several modern ground-based optical instruments have indicated similar systematic differences of their catalogues with respect to the FK5. Thus, the FK5 apparently needs a correction of about $60 \cdot \sin (6 \delta)$ mas for northern hemisphere declinations, other sine corrections of order 20 mas and some equator correction. These errors are the result of systematically erroneous proper motions due to position errors, particularly in old observations.

A typical old instrument, the Pulkovo Struve-Ertel vertical circle, one of the main participants in the FK5, is compared for instrumental errors and their impact on positions with a modern instrument, the Pulkovo photographic vertical circle. Investigations of their divided circles, reading systems, flexures, levels and seasonal change of instrumental systems indicate that neglect and bad account of the errors in earlier observations could lead to the errors of the fundamental system. It is shown that all the instrumental errors up to 50 mas can be investigated if all environmental and electrical values are continuously registered.

Thus, modern instruments can make observations free from any errors of the fundamental system. They can link the system of Hipparcos/Tycho stars with extragalactic objects by observing all celestial objects up to the 18th magnitude and provide within a few years both coordinates and multicolor photometry of the objects with positional accuracy of about 50 mas. The resulting catalogue as an extragalactic reference frame can be used for the ROEMER or another astrometric satellite (see E. Høg, 1994, Contribution at the 'G.Colombo' memorial conference, Padova).

Acknowledgement: the author thanks the American Astronomical Society for financial support. 